\title{
FINAL FOCUS SYSTEM FOR HIGH INTENSITY BEAMS*
}

\author{
E. Henestroza ${ }^{\#}$, F.M. Bieniosek, S. Eylon, P.K. Roy, and S. S. Yu \\ Lawrence Berkeley National Laboratory, Berkeley, CA 94720, USA
}

\section{Abstract}

The NTX experiment at the Heavy Ion Fusion Virtual National Laboratory is exploring the performance of neutralized final focus systems for high perveance heavy ion beams. The NTX final focus system produces a converging beam at the entrance to the neutralized drift section where it focuses to a small spot. The final focus lattice consists of four pulsed quadrupole magnets. The main issues are the control of emittance growth due to high order fields from magnetic multipoles and image fields. We will present experimental results from NTX on beam envelope and phase space distributions, and compare these results with particle simulations using the particle-in-cell code WARP.

\section{INTRODUCTION}

A driver beam is transported in the final focus section through several strong magnetic quadrupoles, and is then allowed to drift ballistically through neutralizing plasma in a low-density (millitorr) gas onto the target. There are nonlinear processes both in the magnetic section as well as in the neutralized transport section. To investigate these phenomena, the Neutralized Transport Experiment (NTX) has begun at LBNL [1]. The experimental setup (Fig. 1) consists of three major sections, a low-emittance potassium gun [2], a magnetic transport section with 4 pulsed quadrupoles [3], and a one-meter long drift section with plasma neutralization [4].

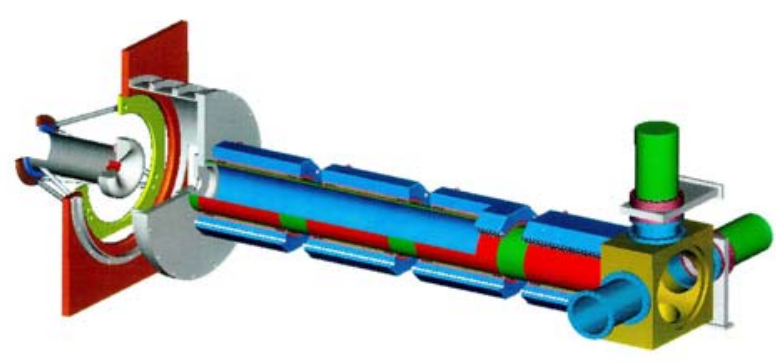

Figure 1: Final focus system for ballistically neutralized drift (cut-away view of components).

The 3-D PIC simulation code WARP3D has been used to design the gun and the magnetic lattice, and predictions

\section{"EHENESTROZA@1bl.gov}

*This work has been performed under the auspices of the US DOE by UC-LBNL under contract DE-AC03-76SF00098, for the Heavy Ion Fusion Virtual National Laboratory. of nonlinear forces have been made and are compared against the NTX experiments. The MRC electromagnetic code LSP has been used to specify the requirements for the plasma source, as well as the predicted degree of neutralization as a function of the various beam and plasma parameters.

\section{FINAL FOCUS LATTICE}

The transport section is designed to correspond closely to a prototypical HIF driver final focus channel. It consists of four pulsed quadrupole magnets with short drift regions, including the drift from the source into the channel and the drift into the plasma neutralization chamber. Figure 2 shows the horizontal and vertical beam envelopes through the system, with and without beam neutralization after the final focus lattice. The quadrupole fields are chosen to obtain a beam of one-meter focal length $(20 \mathrm{~mm},-20 \mathrm{mr})$ at the entrance to the neutralization region.

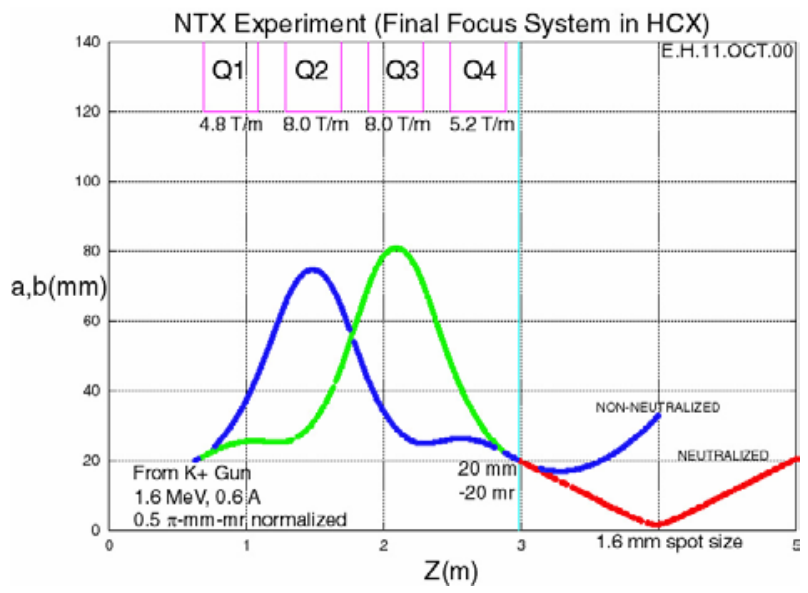

Figure 2: Final focus lattice for ballistically neutralized drift.

The heavy ion beam is space charge dominated in the final focus system, where it has a large cross section and is highly non-paraxial. The beam expands in the two center magnets, which determines the amount of nonlinear magnetic fields that the beam samples as well as the degree of non-paraxial motion. Therefore, particle tracking simulations require the knowledge of the multipole field content of the magnetic field. It can be shown that to second order [5] the main contribution to the beam dynamics come from the normal quadrupole, the normal sextupole and the pseudo-octupole components of the magnetic field. These three components are included in all the numerical simulations performed for the design of the lattice. 


\section{TRANSPORT IN FINAL FOCUS SYSTEM}

A high brightness ion beam is an essential component of the Neutralized Transport Experiment (NTX). An ion beam extracted from a Pierce-type diode suffers from spherical aberrations as evidenced from phase space distortions (high emittance) and from non-uniform density profiles. Since the source of these aberrations are the high order field components, the particles at the edge of the beam are the most affected. One way to generate high brightness beams is to remove the edge of the beam after it is generated in the diode [2]. To study beam transport through the final focus system we have used a $300 \mathrm{keV}$, $25 \mathrm{~mA}$, beam with a normalized emittance of 0.05 $\pi-\mathrm{mm}-\mathrm{mr}$ and a uniform density profile. Optical scintillators imaged by CCD cameras and mechanical slit scanners [6] were used to measure the beam profiles and phase space distributions of the beam at the end of the final focus lattice. Figure 3 shows the good agreement between the measured (top) and calculated (bottom) beam profile and phase space distribution for the nominal energy $(300 \mathrm{keV}, 25 \mathrm{~mA})$ and quadrupole field configuration; it also shows that the final parameters for the nominal case correspond to that of a beam of the required one-meter focal length $(20 \mathrm{~mm}$ radius and $20 \mathrm{mr}$ convergence).
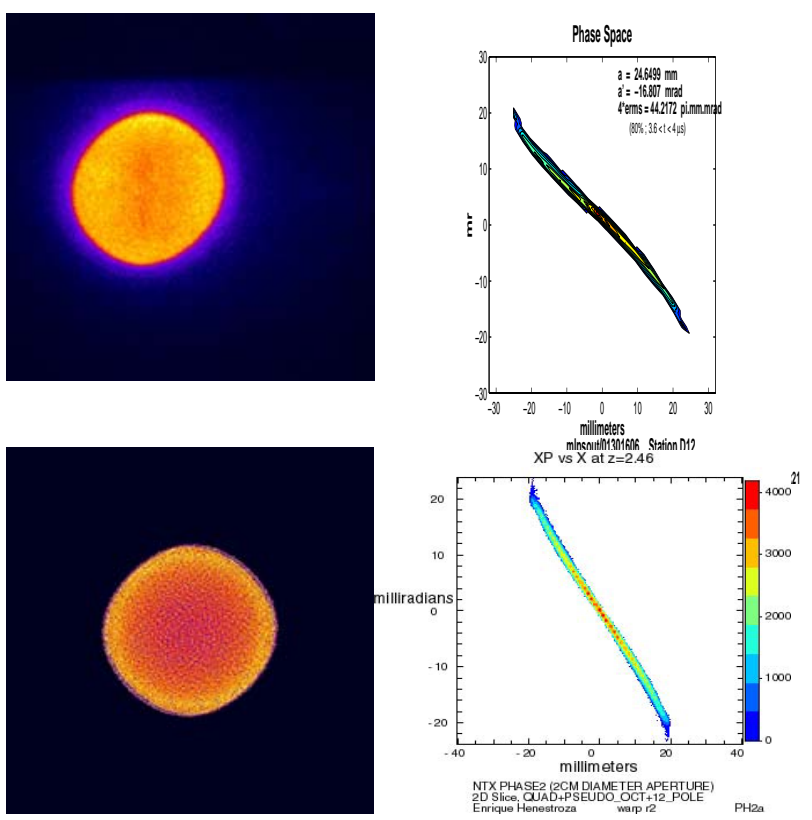

Figure 3: Experimental results and simulations of beam profile and phase space distribution at exit of channel.

The slight distortion of the beam profile was traced back to a small rotation $(5 \mathrm{mr})$ of one of the quadrupoles. The beam is uniform with a narrow rim due to field aberrations and the final normalized emittance of the beam is $\sim 0.1 \pi-\mathrm{mm}-\mathrm{mr}$ as required by the plasma neutralization experiment [4].
The low emittance ( $30 \pi$-mm-mr unnormalized) allows for the beam to be focused to a small spot size (1-2 mm radius) at the focal point for neutralized ballistic transport. Furthermore, Faraday cup measurements of the beam current at entrance and exit of the final focus system have shown insignificant beam loss along the transport channel.

\section{ENERGY AND QUADRUPOLE FIELD SCANS}

We have also performed a complete characterization of the quadrupole lattice by comparing experimental results with particle simulations using the particle-in-cell code WARP. Figure 4 shows the good agreement between the calculated (top) and measured (bottom) beam profiles at the exit of the final focus system when the beam energy is varied in steps of $3 \%$ around the nominal energy (image at center). The numerical simulations track the beam behavior as the beam expands by a large factor when the energy changes from $-9 \%$ to $+9 \%$ around the nominal energy.

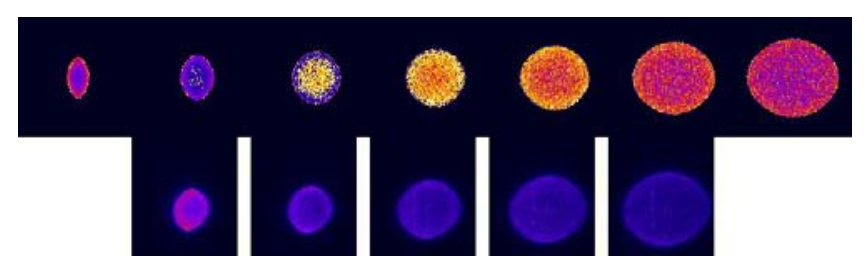

Figure 4: Numerical results and camera images of beam profiles as function of energy.

We also compared (Fig. 5) the beam profiles for several quadrupole strength configurations as calculated by WARP and as measured. For each quadrupole, the corresponding row shows the profiles for a change of $5 \%, 0 \%$ and $+5 \%$ from the nominal quadrupole strength. This comparison shows the good agreement that we have obtained so far, between measurements and simulations.
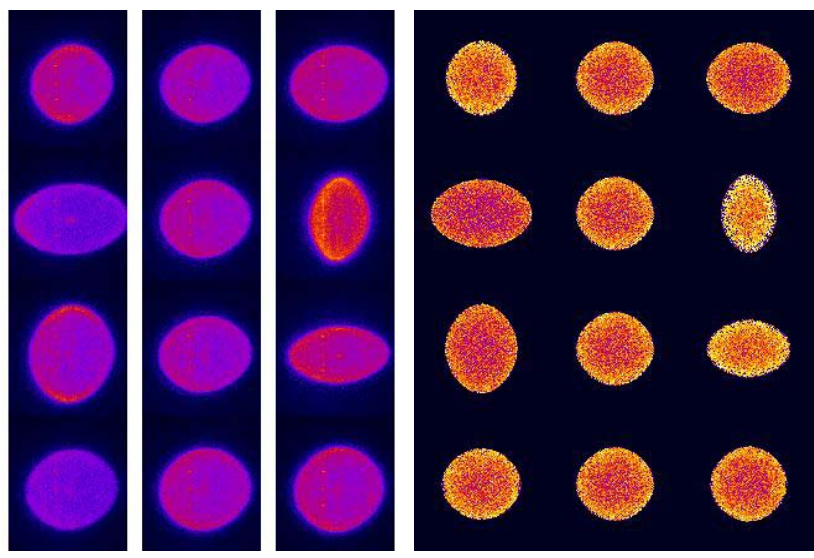

Figure 5: Numerical results and camera images of beam profiles as function of quadrupole field configuration. 


\section{4-D PHASE SPACE MEASUREMENT}

We are developing a new technique to measure the 4-dimensional phase space distribution of the beam using the beam imaging diagnostics [6]. The phase space distribution $\mathrm{f}\left(\mathrm{x}, \mathrm{y}, \mathrm{x}^{\prime}, \mathrm{y}^{\prime}\right)$ can be measured by scanning the beam with a small pinhole (20 mils in diameter) and letting the transmitted beamlet to travel a long distance ( $\sim 1$ meter) before striking the scintillator where an image is taken. The position of the pinhole defines the coordinates $\mathrm{x}$ and $\mathrm{y}$, and from the image we can extract the density distribution of $x^{\prime}$ and $y^{\prime}$. Due to the fact that the beam at the exit of the final focus system is prepared to focus to small spot in the absence of space charge, a standard pepperpot technique does not work since all the individual beamlets would fall on a single spot. Figure 6 shows schematically this technique along with some images of the individual beamlets that show a very detailed structure of the phase space distribution. The knowledge of the 4-dimensional phase space will be essential in order to run more realistic simulations of the beam focusing to a small spot when drifting through neutralizing plasma.

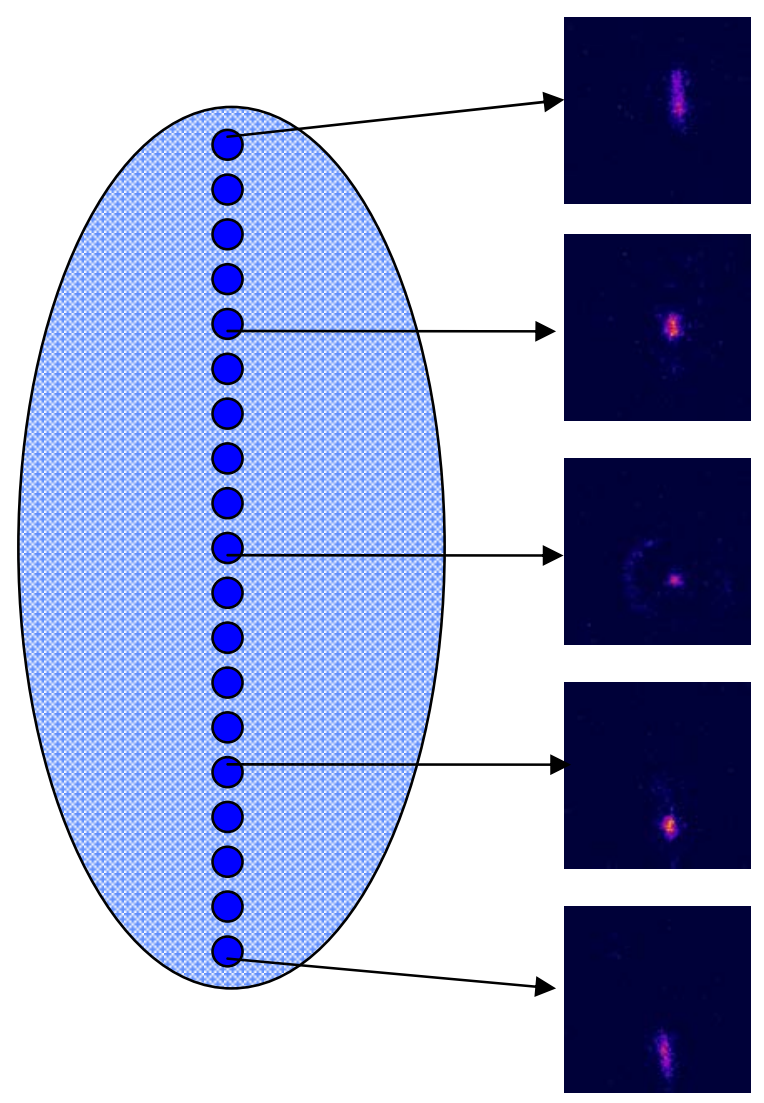

Figure 6: Beam imaging technique to measure the phase space distribution $\mathrm{f}\left(\mathrm{x}, \mathrm{y}, \mathrm{x}^{\prime}, \mathrm{y}^{\prime}\right)$.

\section{CONCLUSIONS}

Initial experiments of beam transport through the NTX final focus systems has produced the design beam parameters required for the neutralization transport experiment. Further measurements to obtain the 4-dimensional phase space distribution at the exit of the final focus system will provide the initial condition for realistic simulation of the beam dynamics when ballistically focused through neutralizing plasma.

\section{REFERENCES}

[1] S. S. Yu et al., "Focusing and Neutralization of Intense Ion Beams", Proc. PAC '03, TOAA001.

[2] S. Eylon et al., "High brightness potassium source for the HIF neutralized transport experiment", Proc. PAC '03, WPPG012.

[3] D. Shuman et al., "Magnetic lattice for the HIF neutralized transport experiment", Proc. PAC '03, WPPG017.

[4] E. Henestroza et al., "Neutralized Transport of High Intensity Beams", Proc. PAC '03, WPPG014.

[5] D. Neuffer, "Geometric aberrations in final focusing for heavy ion fusion", Proc. HIF Workshop, ANL, Sept. 19-26, 1978, ANL-79-41.

[6] F.M. Bieniosek et al., "Beam imaging diagnostics for heavy ion beam fusion experiments", Proc. PAC '03, WPPB050. 